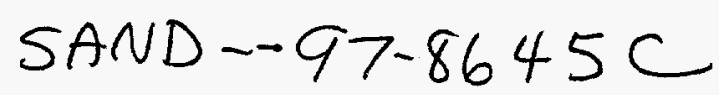

\title{
THE USE OF MAVIS II TO INTEGRATE THE MODELING AND ANALYSIS OF EXPLOSIVE VALVE INTERACTIONS
}

\author{
Raymond Ng and Davina M. Kwon \\ Sandia National Laboratories \\ Livermore, California, USA 94551-0969
}

\section{RECEIVED \\ JUL 211997 \\ OSTI}

The MAVIS II computer program provides for the modeling and analysis of explosive valve interactions. This report describes the individual components of the program and how MAVIS II is used with other available tools to integrate the design and understanding of explosive valves.

The rationale and model used for each valve interaction is described. Comparisons of the calculated results with available data have demonstrated the feasibility and accuracy of using MAVIS II for analytical studies of explosive valve interactions. The model used for the explosive or pyrotechnic used as the driving force in explosive valves is the most critical to be understood and modeled.

MAVIS II is an advanced version that incorporates a plastic, as well as elastic, modeling of the deformations experienced when plungers are forced into a bore. The inclusion of a plastic model has greatly expanded the use of MAVIS for all categories (opening, closure, or combined) of valves, especially for the closure valves in which the sealing operation requires the plastic deformation of either a plunger or bore over a relatively large area.

In order to increase its effectiveness, the use of MAVIS II should be integrated with the results from available experimental hardware. Test hardware such as the Velocity Interferometer System for Any Reflector (VISAR) and Velocity Generator tests provide experimental data for accurate comparison of the actual valve functions. Variable Explosive Chamber (VEC) and Constant Explosive Volume (CEV) tests are used to provide the proper explosive equation-ofstate for the MAVIS calculations of the explosive driving forces. The rationale and logistics of this integration is demonstrated through an example.

A recent valve design is used to demonstrate how MAVIS II can be integrated with experimental tools to provide an understanding of the interactions in this valve.

\section{INTRODUCTION}

Explosively actuated valves can be categorized based on one of three different functions: explosive valves can (1) open a transfer path, (2) close a transfer path or (3) perform a combination of opening a path while closing another one. Within each category are unique features that need to be understood and designed in order to improve our present valves and provide consistently reliable valves for future complex needs.

The MAVIS ${ }^{1}$ computer program was developed for the modeling and analysis of valve interaction $\underline{\underline{s}}$ in explosively actuated valves. It provides the design engineer with an analytical tool to be used for every aspect of the understanding, design and analysis of explosive valves. MAVIS II includes an elastic-plastic model ${ }^{2}$ to handle the calculation of plastic deformation. This was especially useful for closure valves in which extensive plastic deformation of either a plunger or housing is required in order to produce an adequate seal.

A design tool like MAVIS II is best used when integrated with available empirical tools. Test hardware such as the Velocity Interferometer System for Any Reflector (VISAR) and the Velocity Generator provide experimental data used to verify and improve the modeling in MAVIS II. Other test hardware, the Variable Explosive Chamber (VEC) and Constant Explosive Volume (CEV), provide the data to calculate the explosive equation of state. 


\section{DISCLAIMER}

This report was prepared as an account of work sponsored by an agency of the United States Government. Neither the United States Government nor any agency thereof, nor any of their employees, makes any warranty, express or implied, or assumes any legal liability or responsibility for the accuracy, completeness, or usefulness of any information, apparatus, product, or process disclosed, or represents that its use would not infringe privately owned rights. Reference herein to any specific commercial product, process, or service by trade name, trademark, manufacturer, or otherwise does not necessarily constitute or imply its endorsement, recommendation, or favoring by the United States Government or any agency thereof. The views and opinions of authors expressed herein do not necessarily state or reflect those of the United States Government or any agency thereof. 


\section{DISCLAMMER}

Portions of this doeconent may be illegible in electronic imige products. Images are produced from the best available origion docmenert 
A recent valve design is used to demonstrate the use of these tools. MAVIS II calculations are integrated with the results from VEC and VISAR tests to provide an understanding of the unique features of this valve.

\section{EXPLOSIVE VALVES}

There are three types of explosively actuated valves. Representatives of each are shown in Figure 1 and described as follows:

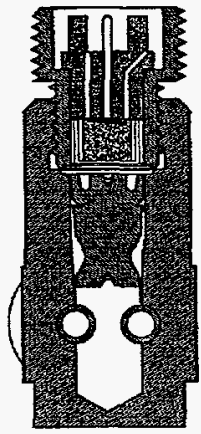

Open

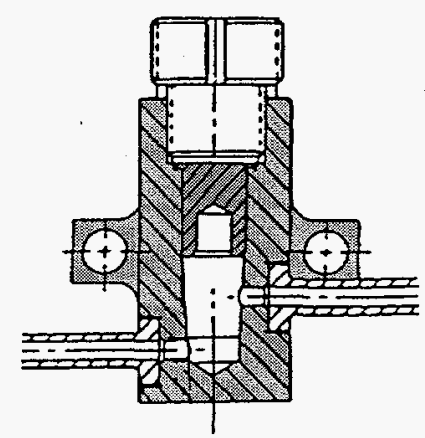

Close

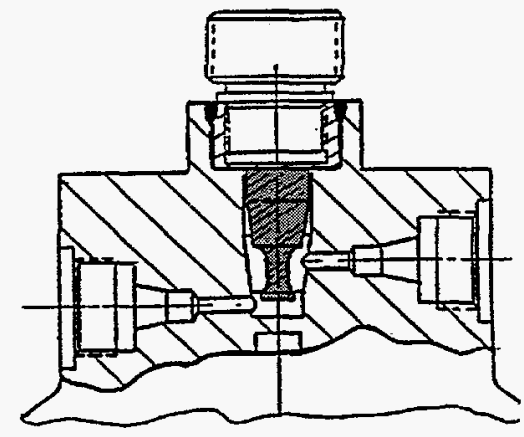

Combination

Figure 1: Three Types of Explosive Valves

(1) The explosive valve can OPEN a transfer path. The example shown in the figure is commonly known as a Mini-valve. A 1.6 gram plunger is driven by the explosive gas down a bore in the housing. As it contacts either one or two $1 / 8^{\prime \prime}$ diameter tubes, the plunger cuts the tube(s), opening a transfer path through the tube(s). The plunger is then stopped by a tapered section of the bore.

(2) The explosive valve can CLOSE a transfer path. The example is a hollow plunger that is driven by the explosive into a tapered section of a bore that contains a through hole used for transfer of a gas. The plunger undergoes sufficient plastic deformation to seal the transfer hole.

(3) The explosive valve can perform a COMBINATION of opening a path while closing another one. The third example is a valve plunger that is driven to first punch through a bulkhead to open a transfer path. It then continues until it is stopped by a tapered section of the bore. A through hole is closed as the plunger deforms into the taper.

These examples are just three representatives of all explosive valves. Many other valves may have significantly different design, size or shape. However, they functionally fall into one of these three categories.

\section{THE MAVIS II MODELING OF THE VALVE INTERACTIONS}

The operational sequence of the previously mentioned Mini-valve is shown in Figure 2 as an example of a typical valve design. The operation of a valve begins with the igniting of the explosive charge. The explosives used in present actuators are forms of different gas generating pyrotechnics. The resulting gas pressure builds up as the charge is burned or detonated, until it exerts sufficient force on the disc to shear it. The gas pressure from the explosive then pushes the plunger (and disc) down the bore of the housing. As the plunger travels down the bore, various interactions will occur.

First, as the plunger moves, the explosive gas will occupy a larger volume and the pressure will decrease accordingly. Second, a drag force will exist between the plunger and 
housing. Third, when the front of the plunger reaches the tubes, a cutting action will take place, causing a resistive shear force to act upon the plunger. Finally, any gas trapped in the housing will push against the plunger, increasing in force as the enclosed housing volume decreases. The valve operation is completed when the plunger is forced to a stop.

Analytical models were developed for every interaction. Some of the more important features of each function in MAVIS II are summarized in the following sections:

\section{Explosive Equation of State}

The equation of state used for the explosive is a modified version of the Jones-Wilkins-Lee (JWL) equation, an empirical description of the adiabatic expansion of a

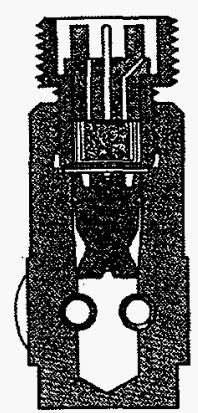

Before

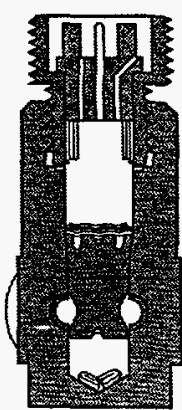

After

Figure 2: Explosive Valve Operational Sequence gaseous product. This model was chosen on the basis of its demonstrated ability to accurately model the region of large expansions typical of those experienced during valve operations. The original equation is:

$$
P=A\left(1-\frac{W}{R_{1} V}\right) e^{-R_{1} V}+B\left(1-\frac{W}{R_{2} V}\right) e^{-R_{2} V}+\frac{W E}{V}
$$

A modification was incorporated to replace the original empirical constants $A$ and $B$, and energy $E$, (all expressed in MPa) with $\rho \bar{A}, \rho \bar{B}$ and $\rho \bar{E}$, respectively. This allowed for input of specific values through the resulting equation:

where:

$$
P=\rho \bar{A}\left(1-\frac{W}{R_{1} V}\right) e^{-R_{1} V}+\rho \bar{B}\left(1-\frac{W}{R_{2} V}\right) e^{-R_{2} V}+\frac{\rho W \bar{E}}{V}
$$

$$
\begin{aligned}
P & =\text { pressure, } \mathrm{MPa} \\
V & =\text { volume ratio (dimensionless) } \\
\bar{E} & =\text { specific energy, } \mathrm{MJ} / \mathrm{kg} \\
\bar{\rho} & =\text { density, } \mathrm{kg} / \mathrm{m}^{3} \\
\bar{A}, \bar{B} & =\text { empirical coefficients, } \mathrm{MJ} / \mathrm{kg} \\
R_{1}, R_{2} & =\text { empirical constants (dimensionless) }>\text { typically } R_{1} \approx 4, R_{2} \approx 1 \\
W & =C_{p} / C_{v}-1 \text { at large expansions (dimensionless) }>\text { typically } 0.2 \leq W \leq 0.4 \\
C_{n} / C_{v} & =\text { ratio of specific heats at constant pressure and volume, respectively }
\end{aligned}
$$

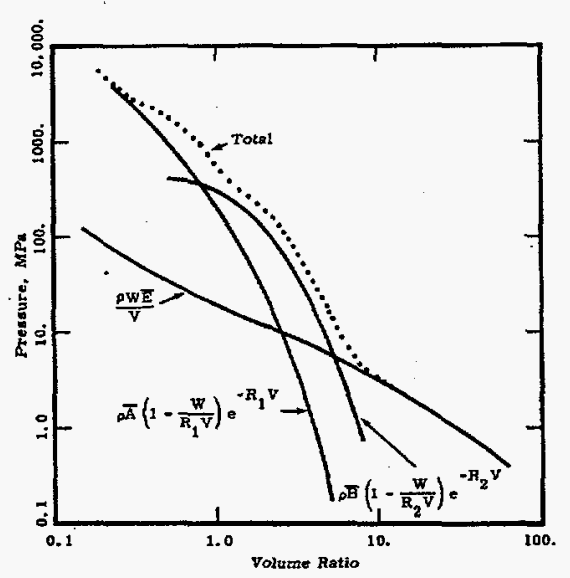

Figure 3: JWL Equation
Each of the terms in the equation would contribute its maximum relative effect at a different volume. Figure 3 shows how the three terms typically contribute to the total pressure.

In addition to the equation of state, another parameter used in the modeling of an explosive is its burn rate. In general, with the ignition of an explosive, the ignition front travels at a velocity which begins at zero and increases to a stable value. This steady state velocity may be either a detonation velocity (if the explosive goes into detonation, producing a shock front) or a deflagration velocity (if the explosive simply continues to burn slow enough as to not 
produce a shock front). While both the detonation and deflagration velocities may be stable, the existence of each is determined by many factors, some of them being the configuration of the explosive, its confinement, pressure, temperature, and physical factors such as mix ratio or grain size.

The steady state burn velocity is difficult to determine. The transient during burn front acceleration is even more difficult to specify. Figure 4 shows two velocity-time curves, one going into detonation and another remaining at a lower deflagration velocity. For the purpose of the model, the burn front is accelerated uniformly to its final constant velocity. This is an approximation, for it is known that for most explosives the burn rate will increase as a function of pressure. Therefore, another burn rate option would most likely be more accurate than a linear velocity-time model. Three other simple curves are shown in Figure 5. At present, the linear model is the only one available in MAVIS II.

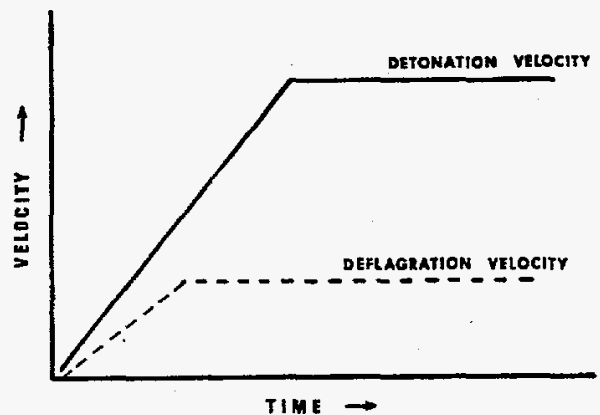

Figure 4: Burn Velocities

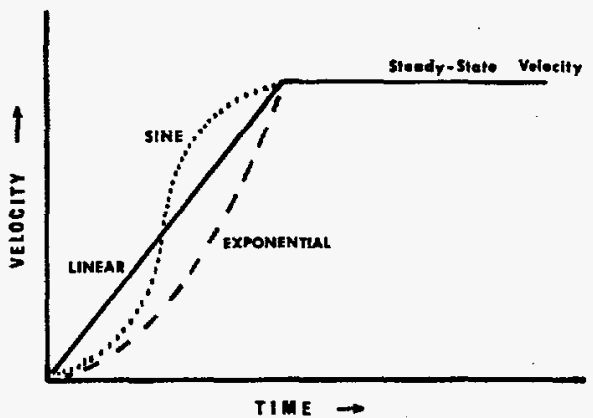

Figure 5: Burn Rate Options

\section{Disc Interactions}

The presence of a disc between the explosive actuator and the plunger temporarily contain the gaseous build-up of explosive pressure, thus delaying the initial motion of the plunger. Because of the delay, once the disc is sheared, the pressure acting upon the plunger can be greater than what would be experienced with no disc. The selection of a disc will cause varying effects, based upon its density, thickness, shear diameter, and shear resistance. A disc design advantageous for one valve design may be harmful for another; all of the interactions must be considered. The MAVIS II program allows for the interactions to be modeled and studied in an analytical way.

The modeling of this part is as follows: The shear force of the disc is first calculated based on the shear thickness, resistance and diameter. Then, as the explosive force is calculated at each time step, no force is allowed to act upon the plunger until the explosive force exceeds the shear force of the disc. Once the disc is sheared, the calculated disc mass is included with the plunger mass in the continuing calculations.

\section{Plunger-Housing Drag Losses}

As the plunger travels down the bore of the housing, there will be a frictional drag along the area of contact. An impact force will also be evident if the plunger is traveling along a tapered region of the bore. These two effects have been modeled into MAVIS by consideration first of the interference between the two parts and then of the magnitude of the forces as caused by the interference.

The ELASTIC interference is easily calculated as the plunger moves along the housing bore by a simple comparison of the radius of the plunger (at various steps along the plunger) with the radius of the housing bore at that point. Therefore, since the location of 
the plunger at any time is known, the interference (or overlap) between the two parts can be incrementally calculated along the length of the plunger.

In order to calculate the forces involved, the plunger is divided into a series of thin discs, each disc having a possible interference fit with an outer ring (representing the housing), as shown in Figure 6. A press- (or shrink-) fit analysis can then be applied to the model to determine the forces involved. The press-fit analysis considers two cylinders assembled in such a way that there is an interference/overlap of the cylinders, resulting in a contact pressure between the two parts. Figure 7 has cylinder 1 inside of cylinder 2 . Since $b_{1}>$ $b_{2}$, a contact pressure exists and a new common radius $b$ is formed.

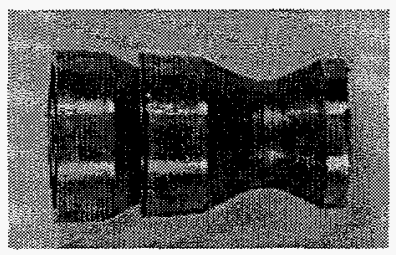

It can be shown ${ }^{4}$ that the increase in internal radius of the outer cylinder (cylinder 2) is:
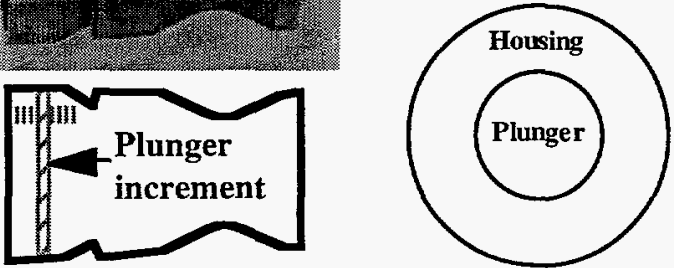

Figure 6: Dividing the

Plunger into Dises

where:

$$
\begin{aligned}
\delta_{2} & =\text { radial increase, } \mathrm{mm} \\
P & =\text { contact pressure, } \mathrm{MPa} \\
E_{2} & =\text { Young's modulus, } \mathrm{MPa} \\
\mu_{2} & =\text { Poisson's ratio } \\
\mathrm{c} & =\text { cylinder } 2 \text { outer radius, } \mathrm{mm} \\
b & =\text { common radius, } \mathrm{mm}
\end{aligned}
$$

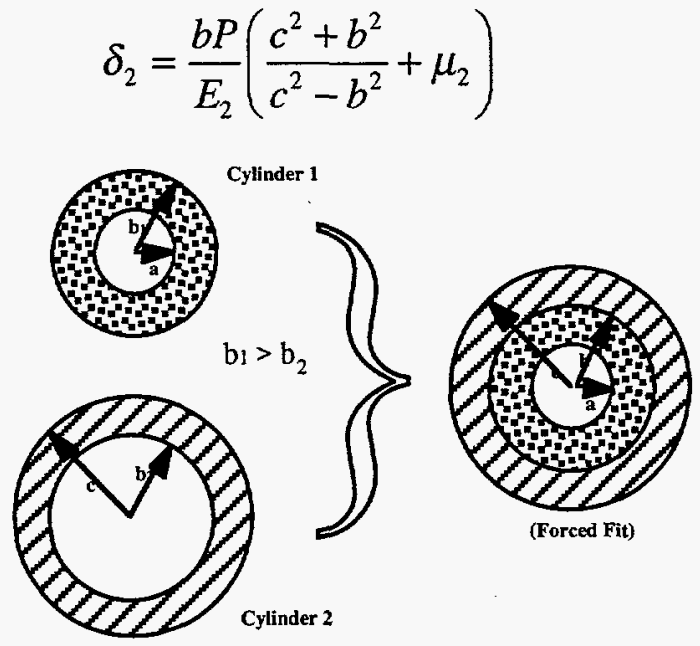

Figure 7: Press-Fit Analysis

Similarly, the decrease in radius of the inner cylinder is:

$$
\delta_{1}=-\frac{b P}{E_{1}}\left(\frac{b^{2}+a^{2}}{b^{2}-a^{2}}-\mu_{1}\right)
$$

where:

$$
\begin{aligned}
\delta_{1} & =\text { radial decrease, } \mathrm{mm} \\
E_{1}, \mu_{1} & =\text { Young's modulus and Poisson's ratio of cylinder } 1 \\
a & =\text { inner radius, } \mathrm{mm}
\end{aligned}
$$

The total elastic interference would be the overlap of the two parts. This is represented by:

$$
\delta=\delta_{2}-\delta_{1}=\frac{b P}{E_{2}}\left(\frac{c^{2}+b^{2}}{c^{2}-b^{2}}+\mu_{2}\right)-\frac{b P}{E_{1}}\left(\frac{b^{2}+a^{2}}{b^{2}-a^{2}}-\mu_{1}\right)
$$

Since the interference $\delta$ is calculated at various increments of the plunger and the dimensions $a, b$ and $c$ and material properties $E$ and $\mu$ are part of the input data, this equation can be solved for the elastic contact pressure $P$.

In the case of plastic deformation occurring in either the plunger or housing, a PLASTIC interference must be calculated. Instead of combining the two concentric cylinders to 
determine a common radius $b$, the cylinders are separated into two different problems of an inner cylinder subjected to an external pressure and an outer cylinder subjected to an internal pressure. The solution to the interference fit lies in superimposing the solutions of the inner and outer cylinders.

The MAVIS II plastic deformation model begins by determining which of four possible scenarios is in effect. These four scenarios are:

- Scenario 1 - Both cylinders remain elastic

- Scenario 2 - The inner cylinder yields while the outer remains elastic

- Scenario 3 - The outer cylinder yields while the inner remains elastic

- Scenario 4 - Both cylinders yield

Solutions for both the pressure and displacement of the inner and outer cylinders under plastic deformation were determined. For the inner cylinder, the relationships were found to be:

$$
\begin{gathered}
p=Y_{i}\left[\ln \frac{c_{i}}{a_{i}}+\frac{1}{2}\left(1-\frac{c_{i}^{2}}{b_{i}^{2}}\right)\right] \\
\left(\delta_{i}\right)_{r=b_{i}}=\frac{\left(1+\mu_{i}\right)}{E_{i}}\left[\left(-p-\frac{Y_{i} c_{i}^{2}}{2 b_{i}^{2}}\right)\left(1-2 \mu_{i}\right) b_{i}-\frac{Y_{i} c_{i}^{2}}{2 b_{i}^{2}}\right]
\end{gathered}
$$

Similarly, for the outer cylinder:

$$
\begin{gathered}
p=Y_{o}\left[\ln \frac{c_{o}}{a_{o}}+\frac{1}{2}\left(1-\frac{c_{o}^{2}}{b_{o}^{2}}\right)\right] \\
\left(\delta_{o}\right)_{r=a_{o}}=\frac{Y_{o}\left(1-2 \mu_{o}\right)}{E_{o}}\left[\frac{3 a_{o}}{2} \ln \frac{a_{o}}{c_{o}}-\frac{3-a_{o}}{4}\left(1-\frac{c_{o}^{2}}{b_{o}^{2}}\right)+\frac{c_{o}^{4}\left(2 \mu_{o}-1\right)}{4 b_{o}^{2} a_{o}}+\frac{5-4 \mu_{o}}{4\left(1-2 \mu_{o}\right)} \frac{c_{o}^{2}}{a_{o}}\right]
\end{gathered}
$$

where:

$$
\begin{aligned}
\delta_{i}, \delta_{o} & =\text { radial displacements of inner and outer cylinders, } \mathrm{mm} \\
p & =\text { contact pressure, } \mathrm{MPa} \\
E_{i}, E_{o} & =\text { Young's modulus of inner and outer cylinder, } \mathrm{MPa} \\
Y_{i}, Y_{o} & =\text { Yield strength of inner and outer cylinder, } \mathrm{MPa} \\
\mu_{i}, \mu_{o} & =\text { Poisson's ratio of inner and outer cylinder } \\
a_{i}, a_{o} & =\text { Inner radius of inner and outer cylinder, } \mathrm{mm} \\
b_{i}, b_{o} & =\text { Outer radius of inner and outer cylinder, } \mathrm{mm} \\
c_{i}, c_{o} & =\text { "Yield radius" of inner and outer cylinder, } \mathrm{mm}
\end{aligned}
$$

MAVIS II will first calculate the elastic pressure (scenario 1) and compare it to the yield pressure for each cylinder. If yielding has occurred, the cylinder with the lowest yield pressure (and therefore the one that yields first) determines the appropriate scenario. Scenario 2 is used if the inner cylinder yields first, and scenario 3 for the outer cylinder being first. The interface pressure from scenario 2 or 3 is then compared to the yield pressure of the cylinder which did not yield. If the second yield pressure is exceeded, then both cylinders have yielded and scenario 4 applies.

For scenario 1 and 2 the interface pressure can be solved directly. In scenario 3 and 4 the pressure and displacement for the outer cylinder are indirectly related as functions of the 
yield radius, $c_{o}$. The solution is obtained by assuming a value for $c_{o}$, then iteratively solving the equations until convergence is reached on the correct interference.

Each elastic or plastic incremental $P$ is calculated and applied over the area $2 \pi b \Delta X$ where $\Delta X$ is the width of the increment along the plunger's length. An incremental contact force is then known. It is from this force that the frictional and impact forces are calculated.

Figure 8 shows a typical increment with a constant pressure $P$. As described, the contact force is calculated for a cylinder with a constant radius. But, in general, there is an angle associated with the contact surface. A force $F_{T}$ (shown in Figure 9) is usually also present.

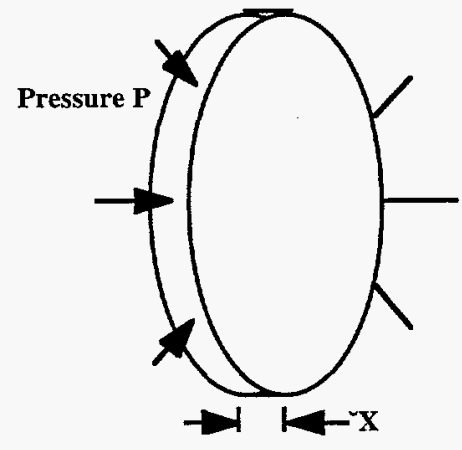

Figure 8: Plunger Increment Under Contact Pressure P

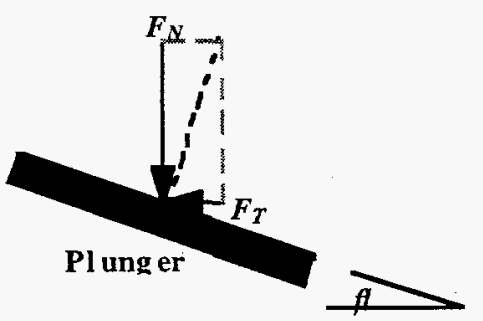

Figure 9: Forces on Surface of Angle $f$
The contact force $F_{N}$ (normal to the direction of travel) is used for the calculation of the frictional force, while the tangential force $F_{T}$ represents the resistive impact force.

The frictional force $F_{f}$ is found to be:

$$
F_{f}=f F_{N}
$$

where $f$ is the coefficient of friction.

Modeling the coefficient of friction involves more than just knowing values of the static and sliding coefficients. Beginning with early scientists like da Vinci ${ }^{5}$ (circa 1500), people have concluded that, for any pair of materials, the static and sliding coefficients of friction were constant. However, those early experiments were carried out under conditions of very small pressures and low speeds. Consequently, the results obtained by them hold only for moderate conditions.

Subsequent investigations by Galton ${ }^{6,7}$ (in 1878) at speeds up to $27 \mathrm{~m} / \mathrm{s}$ and more recent experiments by Bowden and Tabor $^{8}$ and Dokos ${ }^{9}$ at very high sliding speeds (up to 900 $\mathrm{m} / \mathrm{s}$ ) show the coefficient of sliding friction as not constant, but diminishes with speed in a continuous manner. Figure 10 displays a typical curve from Galton's experiments. It is interesting to note that in the region of typical explosive valve velocities $(\sim 100 \mathrm{~m} / \mathrm{s})$, the coefficient of friction is very small. The corresponding frictional force $F_{f}$ would therefore be small. Experiments at very low speeds (i.e., below $0.0003 \mathrm{~m} / \mathrm{s}$ ) have found that the sliding coefficient increases so as to join the value of the static coefficient in a continuous manner. Therefore, the values for the coefficient of friction should be modeled as a function of velocity. The equation used in MAVIS II is:

where:

$$
f=f_{o} e^{-A_{f} V}
$$

$$
\begin{aligned}
f & =\text { coefficient of friction } \\
f_{o} & =\text { static coefficient } \\
V & =\text { velocity, } \mathrm{m} / \mathrm{s} \\
A_{f} & =\text { constant, } \mathrm{s} / \mathrm{m}
\end{aligned}
$$

With this model, the coefficient can be small at speeds at which valves operate and larger for static/slow conditions.

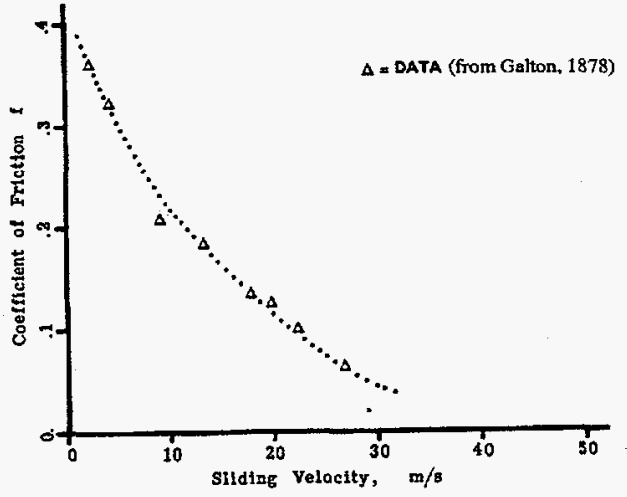

Figure 10: Variation in the Coefficient of Friction as a Function of Velocity 
The impact force, represented by $F_{T}$, may be more significant. Referring again to Figure 9 , once the contact force and the angle of contact are known, the impact force is calculated as:

$$
F_{T}=F_{n} \text { tanf }
$$

The overall resistive force would be the sum of the frictional drag $F_{f}$ and impact $F_{T}$ forces:

$$
F_{\text {Total }}=F_{f}+F_{T}=f F_{N}+F_{N} \tan f l=F_{N}(f+\tan f)
$$

Each increment along the plunger is checked for a resistive force. Once calculated for each zone, the forces are summed to obtain the total resistive force due to the interference between the plunger and housing. In such a process, variations in the contours of the plunger and housing are adequately taken into consideration. The result is a realistic and accurate time history calculation of this resistive force.

\section{Tube Cutting}

As the plunger contacts the tubes, a shearing action takes place. Figure 11 shows a tube chip sheared from the tube by the plunger. A visual unrolling of the sheared surface is approximated by the area enclosed by two concentric ellipses, also shown in Figure 11. The model used for the resistive tube cutting force is:

$$
F_{C}=S_{R} A_{T}
$$

where:

$$
\begin{aligned}
& F_{C}=\text { resistive cutting force, } \mathrm{N} \\
& S_{R}=\text { shear resistance, } \mathrm{MPa} \\
& A_{T}=\text { unsheared area acting against the cutter, } \mathrm{mm}^{2}
\end{aligned}
$$

The sheared area of each tube is analyzed by means of the area on an ellipse. Although this may not be mathematically exact,

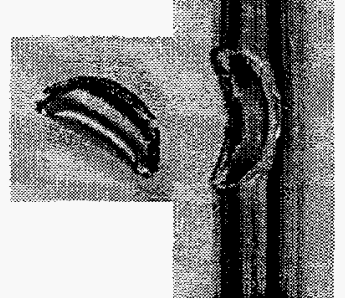

Figure 11: Sheared Tube and its Elliptical Area Approximation the area of an ellipse is a good approximation for the area that is sheared:

$$
A_{E}=, r s
$$

where:

$$
\begin{aligned}
& r=\text { minor axis of ellipse } \\
& s=\text { major axis of ellipse }
\end{aligned}
$$

The axes, $r$ and $s$, are internally calculated for both the inner and outer ellipses that define the sheared area. Minor axis $r$ is based on the location of the plunger with respect to the tube. Figure 12 shows circles (representing the cross section of a tube) intersected by a line (representing the travel of the plunger). The minor axes for the sheared area is calculated once the physical dimensions of the tube, its $x-y$ location and the diameter of the

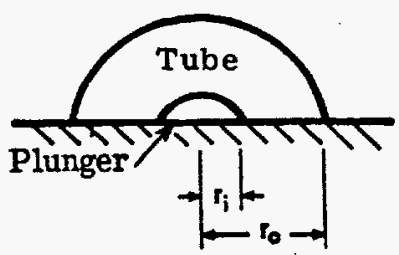

Figure 12: Determining Minor Axis $\mathbf{r}$ plunger are known.

To calculate the major axes, one views the plunger as a cylinder cutting through the side of a tube, as shown in Figure 13. The major axis is half of the length of the arc determined by the tube location. It can also be calculated from the values of tube diameters, location and plunger diameter. For given physical dimensions, a shear area for a tube can be easily calculated. This capability allows for 


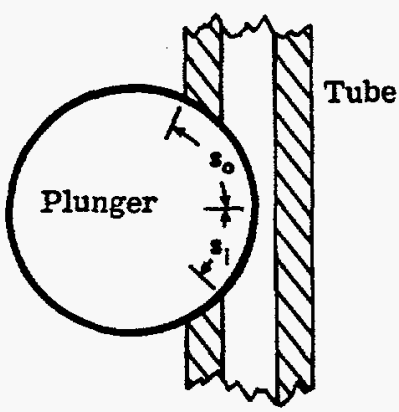

Figure 13: Determining Major Axis s

parametric studies such as the effect that different tube sizes or location would have upon the final plunger stroke.

As the plunger cuts through each tube, the unsheared area $A_{T}$ acting against the plunger changes. The area involved at various stages of the tube cutting is shown in Figure 14 as that area directly under the length in contact with the plunger. The assumption of an elliptical approximation for the area being sheared appears to be valid for this analysis. While values may not be exact for each time step, the overall effect is considered to be, not only an adequate model but, an accurate representation of the actual tube cutting operation.

\section{Internal Gas Pressure}

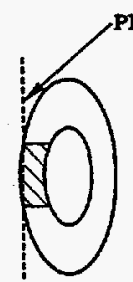

(a)

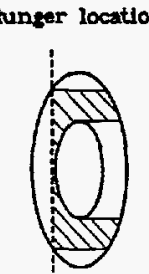

(b)

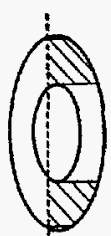

(c)

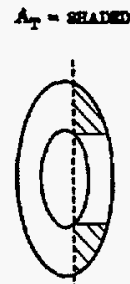

(d)

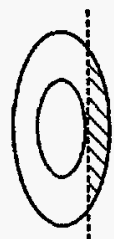

(e)

TIME $t_{a}<t_{b}<t_{c}<t_{d}<t_{c}$

Figure 14: Area Calculations

The volume enclosed by the plunger and housing contains gas at an initial pressure. The pressure within this volume can be large enough to contribute significantly to the slowdown and stopping of the plunger. A sufficiently high pressure can be achieved through either an initial high pressure or a large decrease in the volume. This is modeled in MAVIS II with a perfect gas isentropic process which contributes a load on the plunger.

\section{Temperature Effects}

MAVIS II also includes a temperature effects option which allows for the physical redimensioning of all parts by altering the temperature. This is accomplished through the use of a linear coefficient of thermal expansion for each part. At different temperatures the dimensions for each part can be recalculated and used for the standard interference and volume calculations. The MAVIS II calculations would then proceed in the normal way with the revised dimensions. This option does not yet include any changes to the explosive burn parameters or material properties. However, as it is certain there is an effect, as more research and experiments provide the relationships they can be incorporated into the code.

\section{Initial Velocity}

An initial velocity can be assigned to the plunger at the start of the computational cycles. The explosive equation of state is not needed when this is done. This option allows for the modeling of various experiments (such as tube cutting) in which the plunger is propelled by compressed gas to a stable measured velocity. It also permits an easier parametric study of various effects (such as resistive losses, final stroke and heating) as a function of velocity.

\section{EXPERIMENTAL HARDWARE}

There are various empirical methods that are used to evaluate explosive valves and the explosives and pyrotechnics used as their power sources. A brief description is presented for a few of the more important methods.

\section{Velocity Interferometer System for Any Reflector}

The Velocity Interferometer System for Any Reflector ${ }^{10,11}$ (VISAR) uses a laser beam to track the motion of a surface and measure its velocity. The VISAR uses the interference 
pattern produced by laser light reflected from a moving surface to determine the velocity time history of that surface. The present VISAR hardware and schematic are shown in Figure 15.

Referring to the schematic, the incident laser beam is reflected from the surface of the target, collected, and passed through a polarizer. Any surface motion causes a Doppler shift in the frequency of this light.

The beam is then split, part of

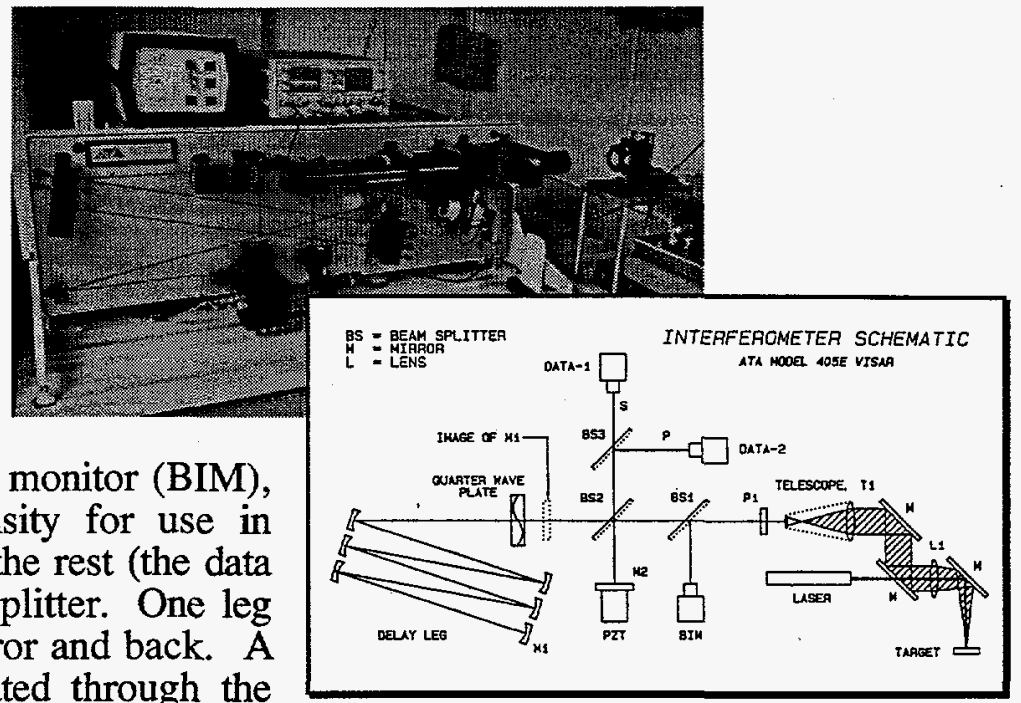

Figure 15: VISAR Hardware and Schematic both legs are combined, the difference caused by the Doppler shift results in the creation of a fringe pattern, with the number of fringes directly proportional to the target's velocity.

The VISAR was first used to measure the velocities of plungers as they were driven by the actuator pressures. A hole would be drilled in the valve housing to expose the inside of the bore. The laser light would be able to enter and exit the bore, providing a non-intrusive way to measure the operation of an explosive valve. An example of a resulting data trace and the calculated velocity history for a Mini-valve are shown in Figure 16.

Other applications of a VISAR for explosive valve testing will be discussed in some following sections of this report.

\section{Velocity Generator}
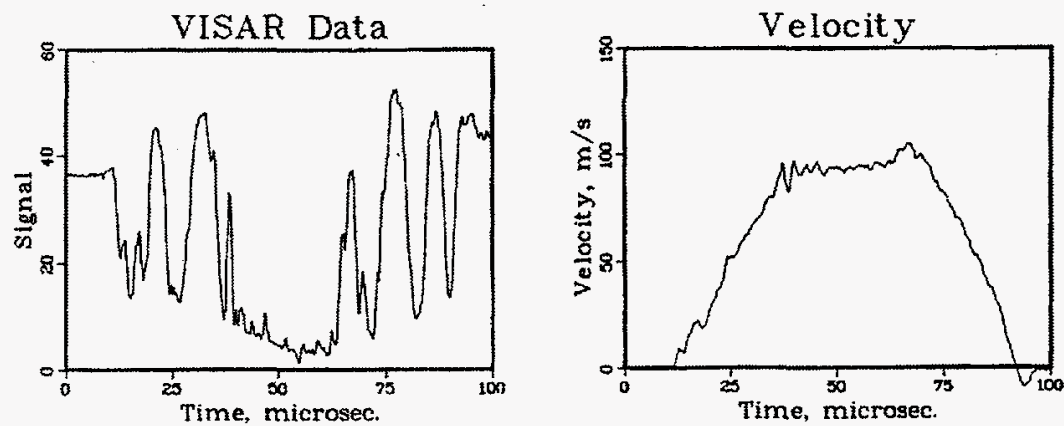

Figure 16: VISAR Measurement of Valve Velocity

A Velocity Generator (shown in Figure 17) is used to propel plungers into various targets at known velocities. This hardware allows for parametric tests to be performed, investigating various effects as a result of plunger velocities. Although not involved with explosives, the Velocity Generator is nevertheless the primary method of simulating the direct result of the explosives by providing the kinetic energy to each plunger, an experimental equivalent to the analytical initial velocity in MAVIS II.

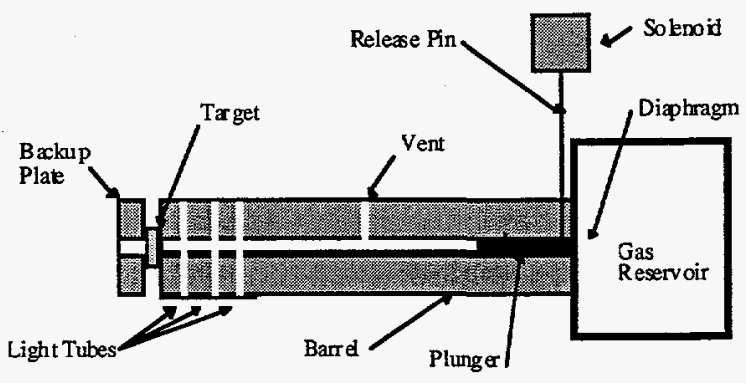

Figure 17: Velocity Generator 
Plungers of different masses can be placed in the barrel of the Velocity Generator. Gas is then pressurized into the Gas Reservoir at different pressures that would result in different velocities once the plunger is released. A pin holds the plunger in place until removed by a solenoid. A vent half-way down the bore released the gas pressure in order to create a freeflying plunger as it enters the target area. The target would consist of any operational function for a valve of interest. Investigations could be performed on cutting of tubes, sealing of transfer holes, punching of various thicknesses of bulkheads, etc.

Once the effects of velocity are known on a desired operation, an explosive output can be specified that would generate the acceptable range of responses.

\section{Variable Explosive Chamber}

The Variable Explosive Chamber ${ }^{12}$ (VEC) test hardware is shown in Figure 18. It was designed and developed to acquire the pressure output relationship of explosives and pyrotechnics used in explosive actuators. Since the actuator is the sole power source for the valves, it is important to know both quantitatively and qualitatively the pressure output characteristics of the explosives.

The basic hardware consists of a housing, a pressure transducer installed in the housing, a slider (into which an explosive actuator can be mounted facing the transducer) press-fitted into a

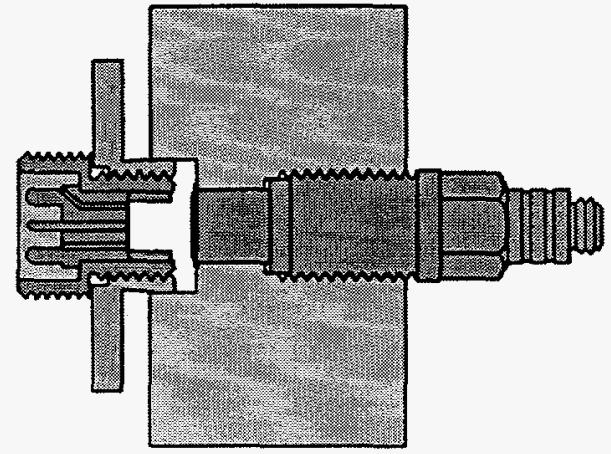

Figure 18: Variable Explosive Chamber (with Actuator/Slider assembly in motion) bore of the housing, and a displacement measuring device (the earlier versions used a Velocity Interferometer System for Any Reflector (VISAR), the present system uses an Eddy Current transducer built into the housing).

When the explosive actuator is ignited, the resulting pressure forces the actuator/slider assembly out of the bore. As the assembly is sliding out of the bore, the pressure transducer measures the pressure during this time of increasing volume. Simultaneously a VISAR or Eddy Current transducer measures the slider's velocity, which is converted into a volume history. This is combined with the pressure history to generate a pressurevolume equation-of-state for the explosive tested. The main feature of this design is this simultaneous measurement of the pressure and volume histories. It is only through these that resulting pressure-volume equation of states can be determined for each explosive.

An example of the importance of the VEC test is given through its present use as an acceptance test for the explosive actuators used in explosively actuated valves. Figure 19 shows the results from a typical VEC acceptance test in which a VISAR is used to record the velocity information.

The upper two traces are the recorded pressure history and VISAR data fringes respectively. Using the fringes, velocity and stroke histories are calculated and plotted. The pressure history is then plotted versus the stroke to generate the curve shown in the Acceptance Curve.
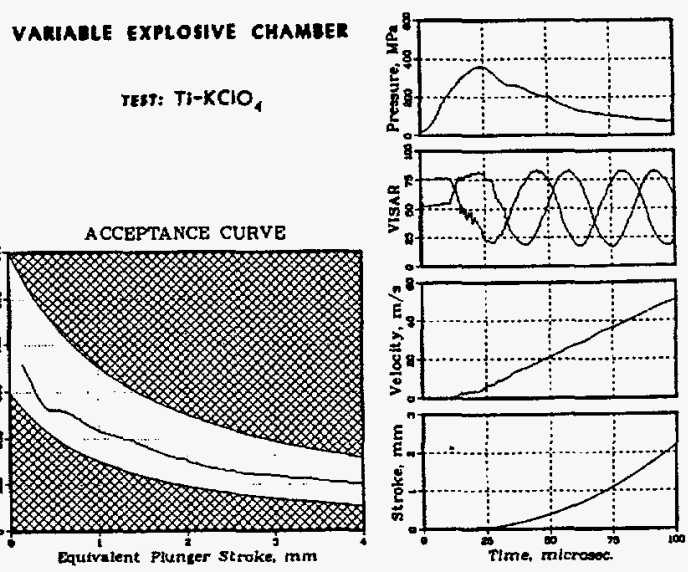

Figure 19: VEC Acceptance Test 
In this example, the acceptance curve displays the required pressure as a function of an equivalent plunger stroke. The plunger stroke can easily be converted into a volume history, thereby creating the pressure-volume relationship of an explosive equation of state.

\section{Constant Explosive Chamber}

The Constant Explosive Chamber (CEV) hardware is a special spin-off of the VEC hardware, in which a stationary version of the VEC test is employed for information regarding the peak pressure developed by each explosive. Figure 20 shows how the actuator is directly mounted in a one-piece housing which also holds the pressure transducer.

The CEV test was originally used to more accurately determine the peak pressure and rise time of an explosive. The pressure data from a VEC test will not show the actual peak pressure or rise time if the actuator/slider assembly begins to move before all of

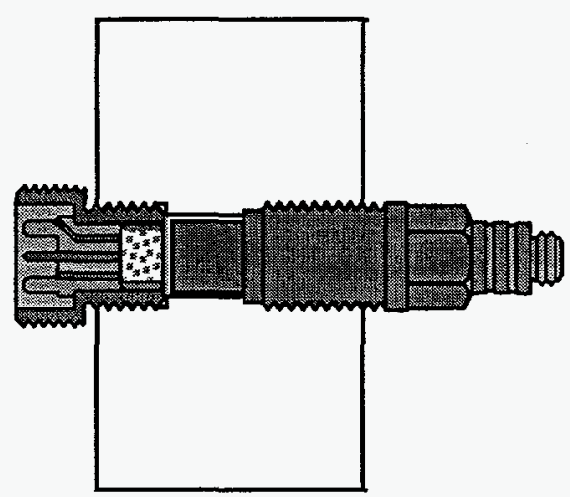

Figure 20: Constant Explosive Volume the explosive has reacted. The use of a CEV is shown in Figure 21 in which two CEV tests (solid lines) are compared with two VEC tests (dashed lines) of identical actuators. With the peak pressure it would be possible to calculate the explosive's equation of state.

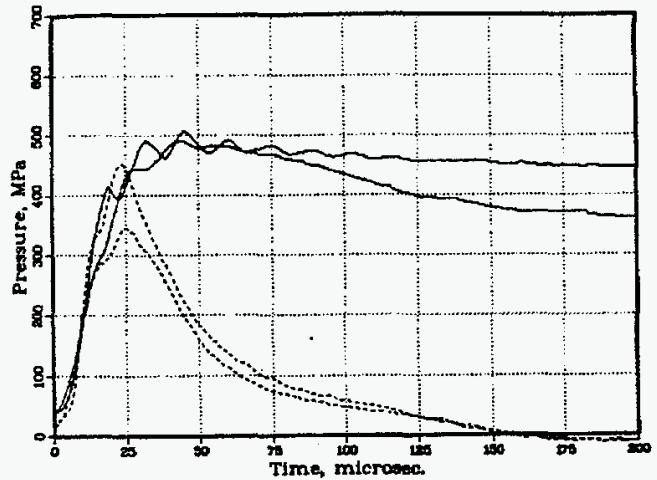

Figure 21: Comparison of CEV Test with VEC Test

zero additional volume) could not sustain its peak pressure. The same actuator design, when fired into an additional 40 cubic millimeters of volume had sufficient mechanical integrity to be self contained.

The CEV has therefore been used (1) to acquire peak pressure information for use with the MAVIS II explosive equation of state modeling, (2) to acquire information about the mechanical integrity of any actuator design, and (3) as an acceptance test for recent actuators.
In addition to generating equation of state information a CEV test is used to investigate the mechanical integrity of various actuators. Recent explosive actuators have the added requirement to remain intact when it is fired into zero additional volume. A mechanical integrity test is formulated with the CEV hardware to make such measurements. Figure 22 shows the type of results that is available with this test. The tested actuator, when fired into itself (i.e.,

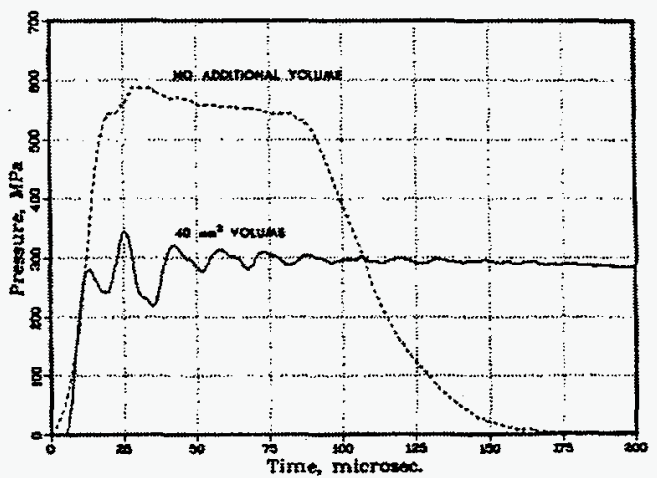

Figure 22: Mechanical Integrity Test

\section{INTEGRATION OF MAVIS II}

As previously stated, a design tool like MAVIS II is best used when integrated with available empirical tools. This section includes a discussion of a recent valve design, 
known as the Striker valve, and the use of MAVIS II and various empirical tools for the modeling and analysis of the explosive valve interactions.

As shown in Figure 23, the Striker valve is basically a Mini-valve that uses two plungers. One plunger is the Mini-valve plunger design and the second is the Striker plunger,

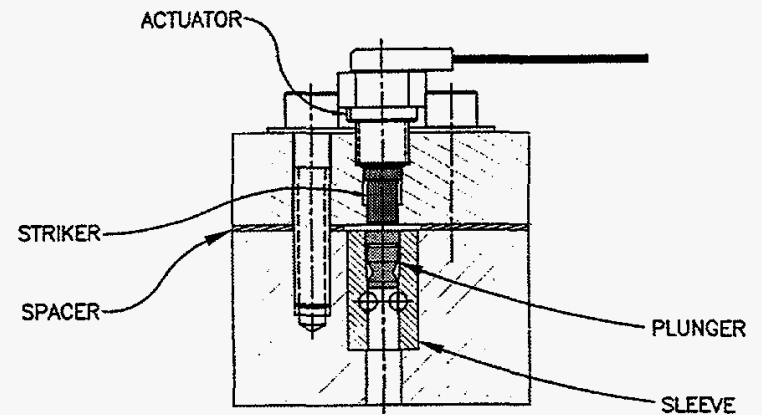

Figure 23: The Striker Valve designed to transfer the energy from the explosive actuator to the Mini-valve plunger. When the actuator is ignited, the pressure will accelerate the Striker plunger down a bore until it hits (i.e., "strikes") the Mini-valve plunger. The unique feature in this design is the function of the Striker plunger to transfer sufficient momentum and energy to the Mini-valve plunger for it to acceptably cut the tubes in the Minivalve. The investigation consisted of two major design issues. The first series of "scoping" experiments were designed to evaluate the adequacy of the design. Following a successful completion of that phase, a second series concentrated on detailed information of the motion of the striker by itself.

The explosive actuator used on this design had been characterized many years ago through the use of the VEC and CEV hardware. This had not only proved the adequacy of the actuator for this valve application but provided the equation of state that would be used for the MAVIS II modeling. Due to the past VEC and CEV data, it was not necessary to use the VEC or CEV for any additional information for this design. The two techniques used to analyze this design were MAVIS II and VISAR. Their integration into the investigation is explained for each phase of the study.

At the beginning of the scoping phase, MAVIS II was used as an aid to the design of the experimental variables. Due to the unique nature of this valve design, the design was separated into two different models for MAVIS II. The first method combined the two plunger into one mass. MAVIS II could then be used in its standard mode of calculations. The second method was to split the responses of the two plungers into two separate set of calculations. The first MAVIS model consisted of the actuator propelling the striker plunger down a bore. The velocity at the given stroke at which the striker would hit the second plunger was then used as an initial velocity for a Mini-valve plunger in the calculations of the second model. This model assumed complete transfer of the momentum from the Striker plunger to the Mini-Valve plunger.

Figure 24 shows the MAVIS II results for one of the configurations tested during the scoping phase of the experiments. (The second method of modeling within MAVIS will be illustrated in the discussion of the second phase of tests.) As shown in the plot, a peak velocity of $80 \mathrm{~m} / \mathrm{s}$ and total stroke of $5.8 \mathrm{~mm}$ were predicted.

The testing of the scoping phase was then performed using the VISAR to record the velocity and displacement of the Mini-valve plunger. In general, the tests and the use of the VISAR were successful. The VISAR data signals and resulting calculations of velocity and stroke are shown as Figure 25 and Figure 26.

MAVIS II: Striker/Mini-Valve

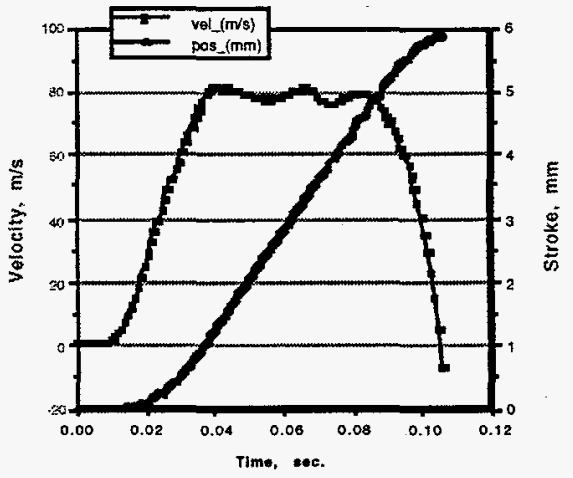

Figure 24: MAVIS Calculations for Striker/Mini-Valve 

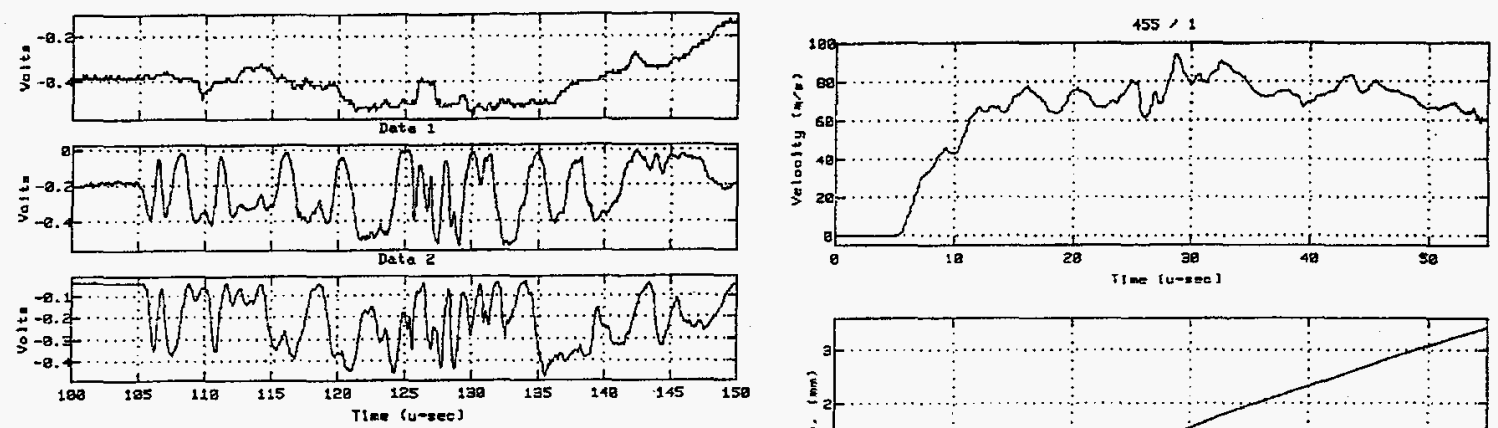

Figure 25: VISAR Data for Striker/Mini-Valve

The VISAR data signals are very representative of what was measured on each test. All of them had a loss of signal

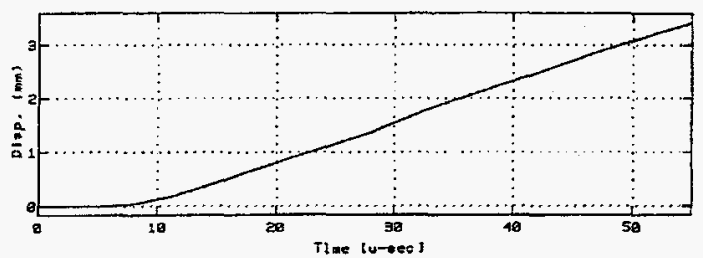

Figure 26: Calculated Striker/MiniValve Velocity and Stroke after approximately 50 microseconds. The most likely explanation is that a tube chip, from the plunger cutting the two tubes, traveled into the path of the laser beam, thereby blocking the data acquisition. However, most of the relevant data had been recorded by then, and the only lost information would be the stopping action of the Mini-valve.

A comparison of the VISAR calculated velocity and stroke with the MAVIS II predictions revealed a very good correlation. Because the laser light was interrupted, the VISAR measurements did not result in a final stroke. However, the post-test measurements on the test hardware showed the final stroke for this test to be $6.06 \mathrm{~mm}$, compared to a MAVIS II calculation of $5.8 \mathrm{~mm}$. The VISAR results confirm the capability of MAVIS II to model the interactions in this unique valve.

The second phase of testing was designed to acquire more detailed information on the motion of the Striker plunger. Therefore, the investigation was just focused on the actuator and striker assembly. MAVIS II calculations were performed with an actuator propelling the Striker plunger down its bore. The calculations of velocity and stroke are shown in Figure 27. Of particularly interest to this specific design is the velocity that would be present at a stroke of $1 \mathrm{~mm}$. This would be the location of impact of the Striker plunger onto the Mini-valve plunger. As indicated by the line on the plot, the Striker velocity at a stroke of 1 $\mathrm{mm}$ is approximately $80 \mathrm{~m} / \mathrm{s}$. This is the velocity that could be used as the initial velocity for a Minivalve plunger in the MAVIS II modeling.

VISAR measurements were also made on the hardware tested in this second phase. The laser beam was incident upon the face of the Striker plunger as it was propelled by the explosive gases from the actuator. VISAR data signals were recorded for each test, and the velocities and displacements calculated. Figure 28 shows the VISAR results for a "Striker Only" condition.

Again, a comparison of the VISAR calculated velocity and stroke with the MAVIS II predictions

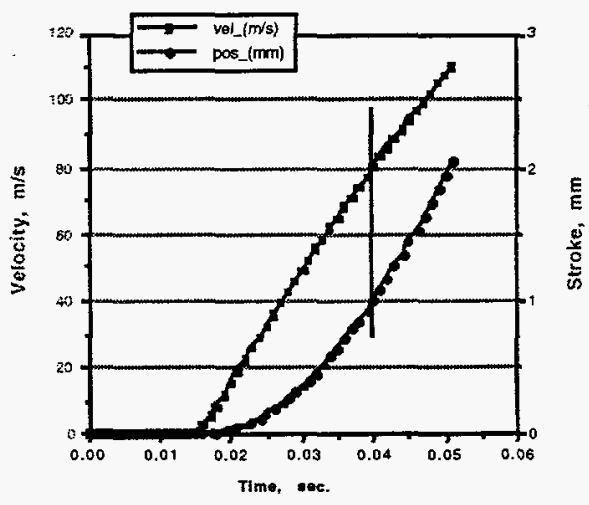

Figure 27: MAVIS. Calculations for Striker Only

reveals very good correlations. Measurements taken on two test hardware show the Striker plunger stroke to be 4.90 and $4.98 \mathrm{~mm}$, as compared to the VISAR calculation of $4.7 \mathrm{~mm}$. 
This is a second verification of the capability of MAVIS II to model these very unique interactions.

MAVIS II was successfully used for the study of this new valve design. This example demonstrated the integration of the modeling with available experimental tools to increase its effectiveness in providing an understanding of the interactions found in this design.

\section{CONCLUSIONS}

The MAVIS II computer program provides an effective tool for the modeling and analysis of explosive valve interactions. It
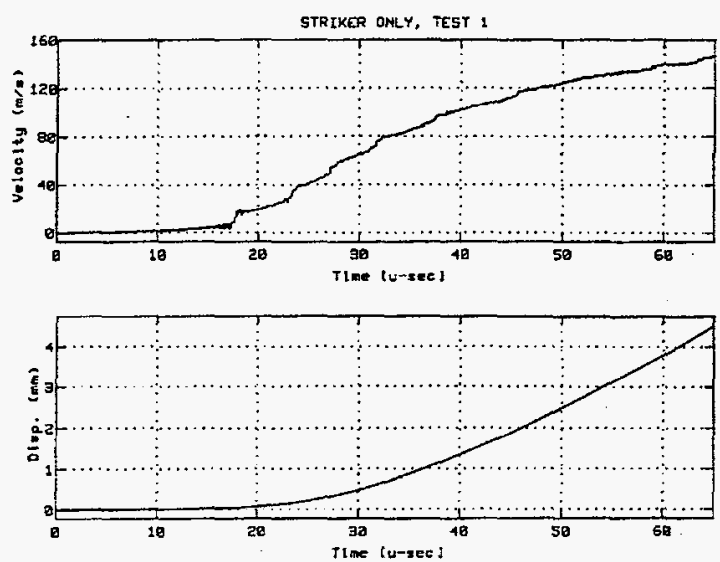

\section{Figure 28: Calculated Striker Only} VISAR Velocity and Stroke

has also been demonstrated that, in order to increase its effectiveness, the use of MAVIS II should be integrated with the results from available experimental hardware. The Striker valve design was used to demonstrate the rationale and logistics of this integration.

MAVIS II has proven itself as an useful model. Empirical tools continue to provide information necessary for its continued application with explosively actuated valves. This is especially true for the modeling of the explosive equation of state and related properties. Future refinements and testing methods will add to the capabilities of this computer program and integration techniques.

\section{REFERENCES}

${ }^{1}$ R. Ng, MAVIS - A Computer Program for the Modeling and Analysis of Explosive Valve Interactions, Sandia Laboratories, Livermore, CA, SAND75-8018, February 1976.

${ }^{2}$ A. F. Emery, B. K. Jones, M. F. Hardwick, and R. Ng, An Investigation of the ElasticPlastic Behavior of Explosively Actuated Valves, Sandia National Laboratories, Livermore, CA, SAND88-8214, July 1988.

3 E. L. Lee, H. C. Hornig, and J. W. Kury, Adiabatic Expansion of High Explosive Detonation Products, Lawrence Livermore Laboratory, Livermore, CA, UCRL-50422, May 1968.

${ }^{4}$ J. E. Shigley, Mechanical Engineering Design, McGraw-Hill Book Co., Inc., New York, 1963, pp. 564-567.

${ }^{5}$ Leonardo da Vinci, Notebooks, Jonathan Cape, London, 1938.

${ }^{6}$ D. Galton, "The Action of Brakes. On the Effect of Brakes upon Railway Brakes," Engineering, V. 25, June 14, 1878, pp. 469-472.

${ }^{7}$ D. Galton, "Railway Brakes. On the Coefficient of Friction from Experiments on Railway Brakes," Engineering, V. 26, August 23, 1878, pp. 153-159.

${ }^{8}$ F. P. Bowden and D. Tabor, Friction and Lubrication of Solids, The University Press, Oxford, 1954.

${ }^{9}$ S. J. Dokos, "Sliding Friction Under Extreme Pressures - 1," Journal of Applied Mechanics, V. 13A, June 1946, pp. 148-156.

${ }_{10} \mathrm{R}$. Ng, VISAR Measurements of Velocities in Explosive Valves, Sandia Laboratories, Livermore, CA, SAND76-8048.

11 M. F. Hardwick, Explosive Valve VISAR Testing, Sandia National Laboratories, Livermore, CA, SAND88-8246.

${ }_{12}$ R. Ng, Variable Explosive Chamber Test Results, Sandia Laboratories, Livermore, CA, SAND78-8784, June 1979.

Work supported by the U.S. Department of Energy under contract DE-AC04-94AL85000. 